\title{
MODELACIÓN HIDROGEOLÓGICA EN UN ABANICO ALUVIAL DE COCHABAMBA-BOLIVIA
}

\author{
Laura Rosales, Oliver C. Saavedra, Waldir Soruco
}

\section{RESUMEN}

Los indicios de sobreexplotación de acuíferos en la región del Valle Central de Cochabamba evidencian la necesidad de tener un mejor entendimiento realizando monitoreo de los niveles piezométricos y configurando un modelo hidrogeológico para evaluar el comportamiento de los acuíferos. Alrededor de un 65\% de agua para consumo humano para la región metropolitana de Cochabamba proviene de aguas subterráneas. El área de estudio se ubica en la zona de El Paso al noroeste del Valle de Cochabamba, considerada como una de las zonas de mayor importancia para la explotación de agua subterránea. El área de estudio se ubica dentro un abanico aluvial conformado por cantos rodados, gravas y arenas. El objetivo de este estudio es actualizar y expandir el monitoreo de niveles de una red de pozos en El Paso, como también entender mejor el comportamiento del flujo subterráneo usando un modelo hidrogeológico. El modelo fue configurado con el paquete MODFLOW y se realizaron mediciones de niveles para calibración y validación de los parámetros. La simulación de los niveles calculados con los observados muestra una raíz media cuadrática (RMS) de $5.69 \mathrm{~m}$. y un coeficiente de correlación de 0.75 . La conductividad hidráulica fue identificada como la más sensible a los resultados. La modelación se realizó en estado estacionario presentando una diferencia positiva en el balance de masas que indica un aporte de entrada al sistema de $26 \mathrm{~m}^{3}$. La dirección de flujo es de norte a sur paralelo a la pendiente de la superficie donde la dirección cambia de golpe en algunos puntos debido a la explotación. Los resultados demuestran que existe un equilibro en el sistema con un pequeño aporte lo que es viable realizar estudios en estado transitorio para identificar los cambios en el balance hídrico. Se ha detectado que no existe sobreexplotación debido al equilibrio que presenta un estado estacionario.

Palabras Clave: Aguas Subterráneas, El Paso, Cochabamba, Modelo Hidrogeológico, Niveles Piezométricos, Estado Estacionario, Flujo Subterráneo.

DOI: $10.23881 /$ idupbo.020.1-4i 